\title{
Controlled study of Pseudomonas cepacia and Pseudomonas maltophilia in cystic fibrosis
}

\author{
G Gladman, P J Connor, R F Williams, T J David
}

\begin{abstract}
In a retrospective study, children with cystic fibrosis who were colonised with Pseudomonas cepacia were compared with a control group who were colonised with Pseudomonas maltophilia. Out of 216 children with cystic fibrosis seen between 1983 and $1990, P$ cepacia was recovered from 13 (median age at colonisation $12 \cdot 2$ years) and $P$ maltophilia from 23 (median age at first colonisation 6.1 years), and both organisms were recovered in five cases. With the exception of two patients with $P$ cepacia in whom no other pathogens were found, all the patients with $P$ cepacia or $P$ maltophilia had co-colonisation with Pseudomonas aeruginosa. The lack of spread of $P$ cepacia to siblings with cystic fibrosis, and the relative lack of inpatient contact between colonised and uncolonised patients suggest that cross infection is not the sole route whereby patients with cystic fibrosis become infected, but the possibility of cross infection cannot be excluded from our data. Three patients with $P$ cepacia died, but two of these had shown appreciable respiratory deterioration before colonisation with $P$ cepacia; there was no evidence of unexpected deterioration in the remainder or in the controls with $P$ maltophilia. By 1990, the prevalence of $P$ cepacia was $9 / 133(7 \%)$ and that of $P$ maltophilia was $13 / 133(10 \%)$, but it was impossible to determine to what extent this increase was due to the introduction of the routine use of selective media. Further studies are required to establish whether patients with and without $P$ cepacia should be segregated.
\end{abstract}

Pseudomonas cepacia is increasingly recognised as an organism that can colonise the respiratory tract of patients with cystic fibrosis, and which may act as a pathogen. The association of $P$ cepacia colonisation with respiratory deterioration in some cases, and the relatively poor response to antibiotic treatment, have caused concern. ${ }^{1}$ Anxiety has been enhanced by the possibility of cross infection between patients with cystic fibrosis. ${ }^{2}$ In an attempt to limit such cross infection, a number of major centres (for example, the Hospital for Sick Children in Toronto; H Levison, personal communication) have recently segregated patients into groups with and without $P$ cepacia colonisation, with separate clinics, isolation, or cohorting during hospitalisation, and rigorous handwashing by professionals after patient contact. Concern about the risk of social contact has led to a number of restrictions imposed by professionals and others (including patients themselves), such as the setting up of 'cepacia' or 'non-cepacia' cystic fibrosis camping holidays. It has been previously claimed in an uncontrolled study that such measures may sharply reduce the number of patients who become colonised with $P$ cepacia ${ }^{3}$ but this has not yet been confirmed by controlled studies, and the possible mode of transmission between patients is obscure.

The microbiology laboratory at Booth Hall Children's Hospital has been storing on a microcomputer data on all bacteria isolated from sputum of patients with cystic fibrosis since 1983. This database has enabled us to perform the present study, the aim of which was to explore possible risk factors for the acquisition of $P$ cepacia colonisation including cross infection. We retrospectively reviewed the clinical and microbiological records of patients with cystic fibrosis, and used as controls those who were colonised with Pseudomonas maltophilia, an organism which we have not hitherto regarded as a pathogen in cystic fibrosis.

\section{Patients and methods}

Between 1983 and 1990, 216 children with confirmed cystic fibrosis have attended the University Department of Child Health at Booth Hall Children's Hospital. As a standard part of our management throughout this period, all patients were instructed to send postal samples of sputum (or cough swabs in those unable to expectorate) to the microbiology laboratory at intervals of two weeks. All patients in whom Pseudomonas aeruginosa was recovered on two or more consecutive sputum samples were treated electively with a two week course of intravenous antipseudomonas antibiotics every three months. Initially all such treatment was given in hospital, but such patients are increasingly treated at home. Oral ciprofloxacin was avoided for such treatment because of the rapid development of resistance. During hospitalisation, sputum samples were collected daily. During the early 1980s, different species of pseudomonas were recognised by close examination of colony appearance, followed by subculture of atypical colonies on selective media. Selective media for $P$ cepacia (blood agar with added ticarcillin and polymyxin B) have been used routinely for all sputum samples since 1988, and the routine use of selective media for $P$ maltophilia (blood agar with added imipenem) in all samples was introduced in 1990.

The following data were sought in this 
retrospective study: age at which $P$ cepacia or $P$ maltophilia was first recovered; prior use of inhaled antibiotics or steroids; details of siblings with cystic fibrosis; number of prior hospital admissions; number of occasions during which a hospital inpatient known to harbour $P$ cepacia or $P$ maltophilia was hospitalised at the same time as a patient from whom $P$ cepacia or $P$ maltophilia had not been recovered but who was to become colonised with either organism within 12 months. Also sought was evidence of unexpected clinical deterioration (defined as an appreciable increase in respiratory symptoms or deterioration in lung function) coinciding with the first recovery of $P$ cepacia or $P$ maltophilia and the therapeutic response to the detection of either organism. Colonisation was defined as a single positive sputum culture.

\section{Results}

$P$ cepacia was recovered from 13 and $P$ maltophilia from 23 children with cystic fibrosis. Both organisms were recovered in five cases. $P$ cepacia was first recovered at 0.3 to $15 \cdot 1$ years (median 12.2), and $P$ maltophilia at 0.8 to 13.6 years (median $6 \cdot 1$ ). With the exception of two patients with $P$ cepacia in whom no other pathogens were found (described below), all of the remaining patients with $P$ cepacia or $P$ maltophilia had co-colonisation with $P$ aeruginosa. The prevalence of $P$ cepacia and $P$ maltophilia recovery are shown in the table. The laboratory also receives specimens from other patients with cystic fibrosis in the North West region, including adults, although these are received on a less regular or frequent basis. Data on prevalence of $P$ cepacia and $P$ maltophilia in all samples is included, for comparison, in the table.

\section{SIBLINGS}

Three out of 13 patients with $P$ cepacia had a sibling with cystic fibrosis, as did 4/23 with $P$ maltophilia. Neither $P$ cepacia nor $P$ maltophilia had been recovered from any of these sibings.

\section{PREVIOUS ADMISSIONS TO HOSPITAL}

The number of previous hospital admissions before the first isolation of $P$ cepacia ranged from 0-19 (median 4.5), compared with 1-14 (median 5) for those with $P$ maltophilia. Two highly atypical children with cystic fibrosis acquired $P$ cepacia during their presenting illness. One presented at 3 months of age with chlamydia pneumonia and failure to thrive. The only bacterial pathogen ever cultured from the respiratory tract was $P$ cepacia, and despite repeated courses of intravenous antibiotics, this child died at the age of 5 months. The other presented at 4 months of age with a six week history of coughing, loose stools and failure to thrive, and a three day history of worsening stridor requiring intubation after a respiratory arrest. $\boldsymbol{P}$ cepacia was grown from blood cultures taken on admission, but no pathogens were isolated from the respiratory tract. This child did not have sickle cell disease, a possible risk factor for $P$ cepacia bacteraemia. ${ }^{4}$ At the age of 19 months he was well, and $P$ cepacia had not been cultured from him again. No patient acquired $P$ maltophilia during the presenting illness.

PRIOR ADMINISTRATION OF INHALED ANTIBIOTICS Seven of the $13(54 \%)$ patients with $P$ cepacia had received twice daily inhaled colistin before the recovery of the organism. Only four of these seven were receiving regular inhaled antibiotics at the time $P$ cepacia was first recovered, and in the other three inhaled colistin had been discontinued at least six months before the first isolation of $P$ cepacia.

Six of the $23(26 \%)$ patients with $P$ maltophilia had received twice daily inhaled colistin or tobramycin before recovery of the organism. Only three (colistin two, tobramycin one) of these six were receiving regular inhaled antibiotics at the time $P$ maltophilia was first isolated, and in the other three (all of whom received colistin) inhaled antibiotics had been discontinued at least six months before the first isolation of $\boldsymbol{P}$ maltophilia. The prior use of inhaled antibiotics was not significantly different between the two groups. $\chi_{1}^{2}=2 \cdot 7$.

PRIOR ADMINISTRATION OF INHALED STEROIDS Two out of 13 (15\%) with $P$ cepacia had received inhaled steroids immediately before isolation of the organism, compared with three out of $23(13 \%)$ patients with $P$ maltophilia.

HOSPITALISATION BEFORE RECOVERY OF P CEPACIA OR P MALTOPHILIA

The 13 patients with $P$ cepacia had between them a total of 120 admissions before the first recovery of the organism, and on five occasions hospitalisation coincided with the presence of at least one other patient with $P$ cepacia on the same ward. The 23 patients with $P$ maltophilia had between them a total of 154 admissions before the first recovery of the organism, but on only one occasion did hospitalisation coincide with the presence of another patient with $P$ maltophilia on the same ward.

Although the number of 'at risk' inpatient

Prevalence (\%) of $P$ cepacia and $P$ maltophilia in respiratory tract secretions in patients with cystic fibrosis for children attending Booth Hall Chilldren's Hospital and for all samples received from patients with cystic fibrosis

\begin{tabular}{|c|c|c|c|c|c|c|}
\hline & \multicolumn{2}{|l|}{1983} & \multicolumn{2}{|l|}{1989} & \multicolumn{2}{|l|}{1990} \\
\hline & Booth Hall & All patients" & Booth Hall & All patients" & Booth Hall & All patients \\
\hline $\begin{array}{l}P \text { cepacia } \\
P \text { maltophilia }\end{array}$ & $\begin{array}{l}0 / 84(0) \\
0 / 84(0)\end{array}$ & $\begin{array}{l}0 / 177(0) \\
0 / 177(0)\end{array}$ & $\begin{array}{l}3 / 124(2) \\
9 / 124(7)\end{array}$ & $\begin{array}{l}16 / 320(5) \\
27 / 320(8)\end{array}$ & $\begin{array}{l}9 / 133(7) \\
13 / 133(10)\end{array}$ & $\begin{array}{l}36 / 331(7) \\
52 / 331(16)\end{array}$ \\
\hline
\end{tabular}

"All patients regularly sending sputum samples to the laboratory, and therefore including those treated at other centres. 
contacts between colonised and those to become colonised in the future was greater in the $P$ cepacia group than in the $P$ maltophilia control group (five $v$ one occasion), in only one of the five $P$ cepacia cases did a 'non-colonised' child in contact with an infected child become colonised with $P$ cepacia within 12 months of the potential contact.

\section{SUBSEQUENT EVENTS}

Of the 13 children with $P$ cepacia, three have died. $P$ cepacia was first isolated six months before their deaths, and two of these cases were showing a considerable clinical deterioration at least 18 months before $P$ cepacia was first isolated, and only in the infant described above, who developed an overwhelming infection, was $P$ cepacia recovery associated with rapid deterioration. Of the remaining 10 children with $P$ cepacia, the time from first isolating the organism to the present time ranges from $0.4-4 \cdot 7$ years (median $1 \cdot 3$ ), and only in one of these patients have we seen a progressive deterioration of clinical condition as assessed by increased sputum production, increased respiratory symptoms, and deteriorating chest radiograph changes.

Of the 23 patients with $P$ maltophilia, all are alive (including the five who were also colonised with $P$ cepacia, and none has shown an unexpected deterioration' in clinical condition since $P$ maltophilia was isolated.

Throughout the study period, our unit has pursued an active policy of regular intravenous anti- $P$ aeruginosa antibiotic treatment every three months in all patients from whom the organism can be regularly recovered. Our policy has always been to discontinue regular intravenous or inhaled antibiotics once $P$ cepacia has been recovered, and avoid intravenous antibiotics unless there was an appreciable respiratory deterioration, in the hope that the cessation of such treatment might be associated with disappearance of $P$ cepacia and recolonisation with $P$ aeruginosa. In three of the 13 patients with $P$ cepacia, the organism ceased to be recoverable from the sputum, and has not been recovered for at least $\mathbf{1 8}$ months after the first isolation.

\section{Discussion}

This report highlights some of the drawbacks of any retrospective study, and there are a number of methodological drawbacks. The patients are a highly selected and therefore atypical group who have attended a cystic fibrosis centre, and thus over represent complex or severe cases and those with particularly well motivated families who are willing to travel large distances for medical supervision. Our examination of acquisition of $P$ cepacia or $P$ maltophilia by cross infection has had to be confined to what may pose the greatest but by no means the sole means of cross infection, namely hospitalisation. The retrospective nature of the study has prevented us from examining other important potential contacts between colonised and noncolonised patients, such as in the outpatient department, the physiotherapy department, hospital organised day trips or social contacts between patients. We have no data on the possibility of environmental contamination. A further problem is the unreliability of the data on $P$ cepacia or $P$ maltophilia colonisation before the routine use of selective media for all specimens, which formerly depended upon the skill and experience of the technician who was looking at culture plates. Also the selective media were introduced at different times for $P$ cepacia and $\boldsymbol{P}$ maltophilia. In addition, although specimens of sputum were sought at intervals of two weeks, there was inevitably some variation in the parents' adherence to this request.

A further difficulty is our poor understanding of the pathogenicity of micro-organisms that can be recovered in the sputum of patients with cystic fibrosis. The fact that bacteria such as $P$ aeruginosa can be recovered from the sputum of patients with cystic fibrosis is no more proof of pathogenicity than the recovery of, for example, Branhamella catarrhalis, the presence of which is usually not reported on the assumption that in this disease it is a normal commensal organism. Although the first recovery of $P$ aeruginosa, or a change from smooth or rough to mucoid colony types, may coincide with respiratory deterioration, it is difficult to find objective evidence of a causal relationship. It is far from clear whether $P$ cepacia, if present in the sputum of a patient with cystic fibrosis, is always or only occasionally a pathogen. ${ }^{5}$ Similarly, we have no objective data to support our assumpton that $P$ maltophilia is not a pathogen in cystic fibrosis.

Despite these limitations, some conclusions can be drawn from our data. It is clear that the prevalences of $\boldsymbol{P}$ cepacia and $\boldsymbol{P}$ maltophilia are increasing in the samples we have received, although it is impossible to determine to what extent this increase is due to the introduction of the routine use of selective media. The lack of spread of $P$ cepacia to siblings, and the frequent absence of inpatient contact with an affected patient before first recovery of the organism, suggest that cross infection is not the sole route whereby patients with cystic fibrosis become infected, but the possibility of cross infection cannot be excluded from our data. Furthermore, others have found that having a sibling with cystic fibrosis and $P$ cepacia colonisation is a significant risk factor for the development of $P$ cepacia. ${ }^{6}{ }^{7}$ Inhaled colistin is a possible risk factor for $P$ cepacia colonisation because most if not all strains of $\boldsymbol{P}$ cepacia are resistant to this agent. The possibility that the use of this agent may encourage the acquisition of $P$ cepacia cannot be discounted, although in the present study the greater use of inhaled colistin before the acquisition of $P$ cepacia compared with $P$ maltophilia was not statistically significant. The published data on the importance of previous antibiotic use are conflicting. In one study, the data suggested a protective effect of aerosolised aminoglycosides, ${ }^{8}$ whereas in another study previous use of aminoglycosides (route unstated) was a risk factor for $\boldsymbol{P}$ cepacia colonisation. ${ }^{6}$ In a third study the previous use of inhaled colistin appeared to have no effect on the risk of $P$ cepacia colonisation. ${ }^{9}$ 
Apart from data on hospitalisation, it was not possible to examine other potential sources of cross infection. Nevertheless, it is of interest that two of the 13 patients who acquired $P$ cepacia were first found to have the organism after successfully initiating home intravenous treatment regimens and reducing contact with the hospital ward. Similarly three of the $P$ maltophilia group were first found to have the organism at least six months after changing from hospital to home for intravenous antibiotic treatment.

Although our data are not supported by objective sequential measurements of disease severity, such as Shwachman scores, radiological scores or lung function test results, the results suggest that the recovery of $P$ cepacia from the sputum is not necessarily associated with a rapid downhill course, although it is undeniable that its presence in a severely affected patient may make antibiotic treatment more complicated and less effective. Other groups have reported either a downhill course ${ }^{1}$ or that an increasing proportion of patients with cystic fibrosis who die are colonised by $P$ cepacia, ${ }^{5710}$ but it is unclear whether there is a causal relationship between colonisation and a worse outcome. In our study, those with $P$ cepacia had a poorer outcome compared with the control group with $P$ maltophilia, but in only two of the 13 cases did we observe an unexpected deterioration, an infant who died at 5 months of age and a boy of 11 years who has had recurrent acute exacerbations of his respiratory problems ever since the first isolation of $P$ cepacia. The majority of our patients, therefore, fit into a category of chronic apparently asymptomatic carriage of $P$ cepacia, and indeed, in some patients where it was clinically possible to suspend anti- pseudomonas treatment for a length of time after first isolating cepacia, we have failed to reculture the organism for up to 18 months.

There is an urgent need for further studies of the possible cross infection of many potential pathogens in cystic fibrosis, including $P$ cepacia and multiple drug resistant strains of $P$ aeruginosa or Staphylococcus aureus. It is regrettable that doctors and patients are currently being obliged to make decisions about segregation and cohorting of patients in the absence of adequate objective published scientific data on the risks or mode of cross infection.

1 Isles A, Maclusky I, Corey M, et al. Pseudomonas cepacia infection in cystic fibrosis: an emerging problem. $\mathcal{f}$ Pediat 1984;104:206-10

2 Lipuma JJ, Dasen SE, Nielsen DW, Stern RC, Stull TL. Person-to-person transmission of Pseudomonas cepacia between patients with cystic fibrosis. Lancet 1990;336: $1094-6$.

3 Thomassen MJ, Demko CA, Doershuk CF, Stern RC Klinger JD. Pseudomonas cepacia: decrease in colonization in patients with cystic fibrosis. Am Rev Respir Dis in patients with

4 Berry MD, Asmar BI. Pseudomonas cepacia bacteremia in hildren with sickle cell hemoglobinopathies. Pediat Infect Dis F 1991;10:696-9.

5 Corey M, Allison L, Prober C, Levison H. Sputum bacteriology in patients with cystic fibrosis in a Toronto hospital. f Infect Dis 1984;149:283.

6 Tablan OC, Chorba TL, Schidlow DV, et al. Pseudomonas cepacia colonization in patients with cystic fibrosis: risk factors and clinical outcome. I Pediatr 1985;107:382-7.

7 Thomassen MJ, Demko CA, Klinger JD, Stern RC. Pseudomonas cepacia colonization among patients with cystic fibrosis. A new opportunist. Am Rev Respir Dis 1985;131: 791-6.

8 Tablan OC and the National Surveillance of $P$ cepacia study group. National surveillance of Pseudomonas cepacia group. National surveillance of Pseudomonas cepacia
colonization of cystic fibrosis patients-an update. Pediatr Pulmonol 1989;4(suppl): 138.

9 Simmonds EJ, Conway SP, Ghoneim ATM, Ross H Littlewood JM. Pseudomonas cepacia: a new pathogen in
Simmonds patients with cystic fibrosis referred to a large centre in th United Kingdom. Arch Dis Child 1990;65:874-7.

10 Tablan OC, Martone WJ, Doershuk CE, et al. Colonization of the respiratory tract with Pseudomonas cepacia in cystic fibrosis. Risk factors and outcomes. Chest 1987;91:527-32. 